\title{
Natural frequency assignment for mass-chain systems with inerters
}

\author{
Yinlong $\mathrm{Hu}^{\mathrm{a}}$, Michael Z. Q. Chen ${ }^{\mathrm{b}}$, Malcolm C. Smith ${ }^{\mathrm{c}, *}$ \\ ${ }^{a}$ College of Energy and Electrical Engineering, Hohai University, Nanjing 211100, China. \\ ${ }^{b}$ School of Automation, Nanjing University of Science and Technology, Nanjing 210094, China. \\ ${ }^{c}$ Department of Engineering, University of Cambridge, Cambridge CB2 1PZ, UK.
}

\begin{abstract}
This paper studies the problem of natural frequency assignment for mass-chain systems with inerters. This is the problem to determine whether an arbitrary set of positive numbers may be assigned as the natural frequencies of a chain of $n$ masses in which each element has fixed mass and is connected to its neighbour by a parallel combination of a spring and inerter. It is proved that mass-chain systems with inerters may have multiple natural frequencies, which is different from conventional mass-chain systems (without inerters) whose natural frequencies are always simple. It is shown that arbitrary assignment of natural frequencies including multiplicities is not possible with the choice of $n$ inerters and $n$ springs. In particular, it is shown that an eigenvalue of multiplicity $m$ may occur only if $n \geq 2 m-1$. However, it is proved that $n-1$ inerters and $n$ springs are necessary and sufficient to freely assign an arbitrary set of distinct positive numbers as the natural frequencies of an $n$-degree-of-freedom mass-chain system.
\end{abstract}

Keywords: Natural frequency assignment, inverse eigenvalue problem, pole placement, passive vibration control, inerter.

\section{Introduction}

The inerter is a two-terminal mechanical device with the property that the applied force at the terminals is proportional to the relative acceleration between them, with the constant of proportionality termed the inertance [1]. One of the principal motivations for the inerter is to achieve a complete correspondence between mechanical and electrical network elements $[1,2]$. An important property of the inerter is that a large inertance can be obtained by devices of relatively small physical mass. As a result, the inerter can used in the control of mechanical systems without adding to the overall mass of the system. Up to now, the inerter has been applied to various systems such as vehicle suspensions $[3,4,5,6]$, train suspensions $[7,8]$, buildings [9, 10], dynamic vibration absorbers [11, 12, 13], vibration isolators [14], landing gears [15], passive mechanical networks [16, 17, 18], etc. The inerter has also been successfully used in Formula One racing since 2005 [2].

\footnotetext{
${ }^{*}$ Corresponding author.

Email addresses: yinlonghu@outlook.com. (Yinlong Hu), mzqchen@outlook.com .

(Michael Z. Q. Chen), mcs@eng.cam.ac.uk. (Malcolm C. Smith)
} 
In this paper, the natural frequency assignment problem for mass-chain systems with inerters is studied, where the adjacent masses are connected by a parallel combination of a spring and an inerter. Mass-chain systems are common mechanical systems, which can be used to describe a variety of mechanical systems such as multi-storey buildings [10, 19], dynamic vibration absorbers [11, 20], vehicle models $[3,4,5,6]$, finite-element models of continuum mechanical systems [21], and so on. Natural frequency is one of the most important inherent properties for mechanical vibration systems, similar to the poles of control system$\mathrm{s}$, determining the dynamic behaviours of mechanical vibration systems. If the excitation frequency is close to one of the natural frequencies, resonance may occur. In practice, it is always desirable to assign the natural frequencies of a vibration system to some specified values or regions such that resonance can be induced [22] or avoided [23]. Therefore, the natural frequency assignment problem for mechanical systems is of practical importance, and it has received much attention $[24,25,26,27]$.

The conventional passive way of achieving natural frequency assignment is by carefully choosing the masses and spring stiffnesses, and it is well known that increasing masses and spring stiffnesses can reduce and increase natural frequencies, respectively. For the mass-chain system where the adjacent masses are connected only by a spring, it has been demonstrated that all natural frequencies are distinct [21], and any $n$ arbitrarily given positive distinct numbers can always be realized as the natural frequencies of an $n$-degree-of-freedom (DOF) system by appropriate choice of the $n$ masses and $n$ spring constants [28]. However, in practice, the masses are normally given with fixed parameters. In such cases the spring stiffnesses alone offer a more limited freedom to adjust the natural frequencies. In [29], it has been demonstrated that if inerters are introduced in parallel to the springs then increasing their inertance can effectively reduce a mechanical systems' natural frequencies. Thus inerters offer a new design possibility in cases where it is not feasible to adjust the masses in a system.

By considering inertances and spring stiffnesses as the design parameters for mass-chain systems with the masses fixed, a fundamental question arises: whether it is possible to realize any arbitrarily given real positive numbers as the natural frequencies of mass-chain systems, and if so, what is the minimal number of inerters required to achieve this. This question will be addressed in this paper by formulating the problem as a direct problem and an inverse problem. The direct problem is an analysis problem, where the multiplicity of a mass-chain system's natural frequencies will be analysed. A difference between mass-chain systems with and without inerters will be demonstrated in that it is possible for mass-chain systems with inerters to have multiple natural frequencies, while the natural frequencies of mass-chain systems without inerters are always simple and distinct. The case of multiple eigenvalues will be studied using a recurrence relation that defines the eigenvalues. It will be shown that there are restrictions on the multiplicities which may occur. In particular it will be shown that an eigenvalue of multiplicity $m$ may occur only if $n \geq 2 m-1$, and necessary and sufficient conditions will be derived for the case of $n=2 m-1$. In contrast, if all the given $n$ positive numbers are distinct, from a synthesis point of view and by using an inverse eigenvalue problem formulation, it will be proved that it is necessary and sufficient to use $n-1$ inerters and $n$ springs to freely assign any arbitrarily given numbers as the natural frequencies of an $n$-degree-of-freedom mass-chain system.

The rest of this paper is organized as follows. Section 2 formulates the natural frequency assignment problem as an eigenvalue assignment problem, and its relation with pole place- 


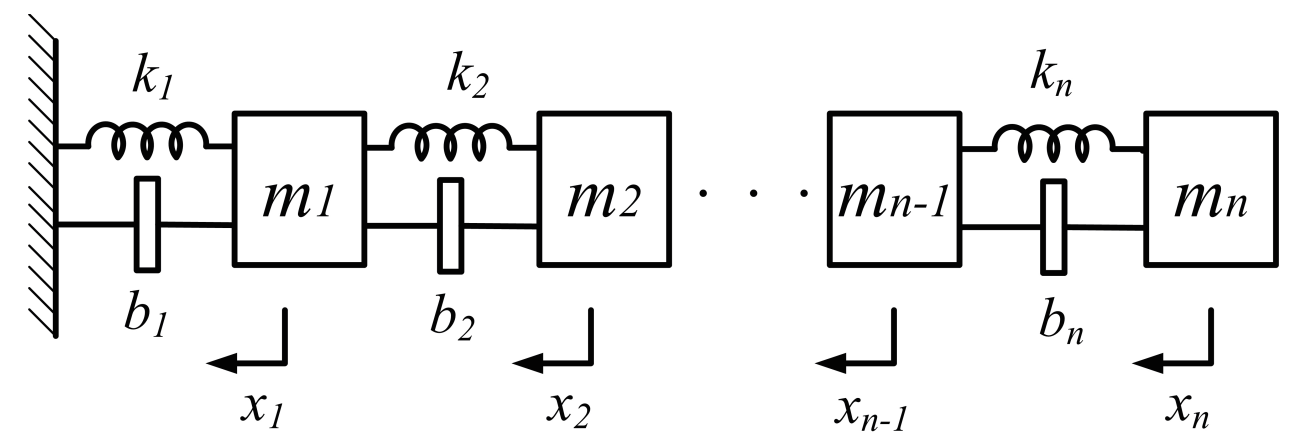

Figure 1: Mass-chain system with inerters.

ment problem is introduced. In Section 3, the direct problem of analyzing the multiplicity of natural frequencies is investigated. Section 4 addresses the natural frequency assignment problem where all the given numbers are distinct. Conclusions are drawn in Section 5.

\section{Problem formulation}

The mass-chain system shown in Fig. 1 is considered. The free vibration equation is

$$
\mathbf{M} \ddot{\mathbf{x}}+\mathbf{K x}=0
$$

where $\mathbf{x}=\left[x_{1}, x_{2}, \ldots, x_{n}\right]^{T}$,

$$
\begin{aligned}
\mathbf{M}= & \mathbf{M}_{0}+\mathbf{B}, \\
\mathbf{M}_{0}= & \operatorname{diag}\left\{m_{1}, m_{2}, \ldots, m_{n}\right\}, \\
\mathbf{B}= & {\left[\begin{array}{ccccc}
b_{1}+b_{2} & -b_{2} & & & \\
-b_{2} & b_{2}+b_{3} & -b_{3} & & \\
& \ddots & \ddots & \ddots & \\
& & -b_{n-1} & b_{n-1}+b_{n} & -b_{n} \\
& & & -b_{n} & b_{n}
\end{array}\right], } \\
\mathbf{K}= & {\left[\begin{array}{ccccc}
k_{1}+k_{2} & -k_{2} & & & \\
-k_{2} & k_{2}+k_{3} & -k_{3} & & \\
& \ddots & \ddots & \ddots & \\
& & -k_{n-1} & k_{n-1}+k_{n} & -k_{n} \\
& & & -k_{n} & k_{n}
\end{array}\right] . }
\end{aligned}
$$

The natural frequencies of the mass-chain system are determined by the square roots of the eigenvalues of the matrix pencil $(\mathbf{K}-\lambda \mathbf{M})$, the eigenvalues being the roots of the following characteristic equation

$$
\operatorname{det}(\mathbf{K}-\lambda \mathbf{M})=0
$$

In the following we will use the terms "eigenvalues" and the "natural frequencies" interchangeably. The following problem is studied in this paper. 
Problem 1. In Fig. 1 let $m_{i}>0(i=1, \ldots, n)$ be given and fixed. Is it possible to choose $k_{i} \geq 0$ and $b_{i} \geq 0$ to realize any arbitrarily given real numbers $0<\lambda_{1} \leq \lambda_{2} \leq \ldots \leq \lambda_{n}$ as the eigenvalues of the $n$-DOF mass-chain system? If so, what is the minimal number of inerters (non-zero $b_{i}$ ) required to accomplish this assignment?

From a control systems point of view, Problem 1 can be viewed as a pole placement problem as follows. Consider the state space model

$$
\dot{y}=\mathbf{A}_{p} y+\mathbf{B}_{p} u,
$$

where $y^{T}=\left[\dot{\mathbf{x}}^{T}, \mathbf{x}^{T}\right]$,

$$
\mathbf{A}_{p}=\left[\begin{array}{ll}
\mathbf{0} & \mathbf{0} \\
\mathbf{I} & \mathbf{0}
\end{array}\right], \quad \mathbf{B}_{p}=\left[\begin{array}{l}
\mathbf{I} \\
\mathbf{0}
\end{array}\right],
$$

and $\mathbf{I}, \mathbf{0}$ denote the identity matrix and zero matrix with appropriate dimensions, respectively. Then, Problem 1 is equivalent to the problem of whether there exists a state feedback $u=\mathbf{K}_{g} y$ with structured state feedback gain matrix in the form of

$$
\mathbf{K}_{g}=\left[\begin{array}{ll}
\mathbf{0} & \mathbf{M}^{-1} \mathbf{K}
\end{array}\right]
$$

such that the eigenvalues of the closed-loop system can be assigned at arbitrary locations on the imaginary axis. It should be noted that Problem 1 requires a special structure for the feedback gain matrix, as well as the constraint that all the design parameters $b_{i}, k_{i}$, $i=1, \ldots, n$ should be real and nonnegative.

In this paper, an inverse eigenvalue problem (IEP) formulation [21, 30] will be employed to address this problem. Note that for the conventional natural frequency assignment problem of using masses and springs and no inerters (i.e. $\left.b_{i}=0, i=1, \ldots, n\right)$, the inertial matrix $\mathbf{M}$ is a diagonal matrix. Then, the problem can be formulated as a Jacobi IEP [21,30]. It is well known that all the eigenvalues are simple (distinct) and any arbitrarily given numbers $0<\lambda_{1}<\lambda_{2}<\ldots<\lambda_{n}$ can be realized as the eigenvalues of the matrix pencil $(\mathbf{K}-\lambda \mathbf{M})$ by carefully choosing the masses and the spring stiffnesses [21, 28]. However, for the problem in this paper, the inertial matrix is a tridiagonal matrix. In this case, the eigenvalues are not always simple, and the Jacobi IEP formulation does not apply, as will be demonstrated in the following sections.

\section{Eigenvalue multiplicity analysis}

In this section it will be shown that, for mass-chain systems with inerters, it is possible to have multiple eigenvalues. The problem of assigning multiple eigenvalues will be discussed.

From (1) and (4), the matrix pencil $(\mathbf{K}-\lambda \mathbf{M})$ is tridiagonal. In Fig. 1, let $m_{i}>0, k_{i}>0$, and $b_{i} \geq 0$ for $i=1, \ldots, n$. Denote

$$
\left\{\begin{aligned}
P_{0}(\lambda) & =1 \\
P_{1}(\lambda) & =k_{1}+k_{2}-\lambda\left(m_{1}+b_{1}+b_{2}\right) \\
P_{2}(\lambda) & =\operatorname{det}\left(\begin{array}{cc}
k_{1}+k_{2}-\lambda\left(m_{1}+b_{1}+b_{2}\right) & -k_{2}+\lambda b_{2} \\
-k_{2}+\lambda b_{2} & k_{2}+k_{3}-\lambda\left(m_{2}+b_{2}+b_{3}\right)
\end{array}\right) \\
& \vdots \\
P_{n}(\lambda) & =\operatorname{det}(\mathbf{K}-\lambda \mathbf{M})
\end{aligned}\right.
$$


Then the sequence $P_{i}(\lambda), i=0,1, \ldots, n$, satisfies the following three-term recurrence relation

$$
P_{i+1}(\lambda)=\left(k_{i+1}+k_{i+2}-\lambda\left(m_{i+1}+b_{i+1}+b_{i+2}\right)\right) P_{i}(\lambda)-\left(k_{i+1}-\lambda b_{i+1}\right)^{2} P_{i-1}(\lambda),
$$

for $i=1, \ldots, n-1$, where $P_{0}(\lambda)=1, P_{1}(\lambda)=k_{1}+k_{2}-\lambda\left(m_{1}+b_{1}+b_{2}\right), k_{n+1}=0$ and $b_{n+1}=0$. The zeros of $P_{n}(\lambda)$ are the eigenvalues of the system. The recurrence relation for the sequence $P_{i}(\lambda)$ makes it a candidate to be a "Sturm sequence".

Definition 1 (Sturm sequence). A sequence $P_{i}(\lambda), i=0,1, \ldots, n$ is called a Sturm sequence in the interval $(a, b)$, if the following two conditions are satisfied [31, Chapter 15]:

1. $P_{0}(\lambda) \neq 0$ for $a<\lambda<b$;

2. for $a<\lambda<b$ and $i=1, \ldots, n-1$, if $P_{i}(\lambda)=0$, then $P_{i-1}(\lambda) P_{i+1}(\lambda)<0$.

Proposition 1. Consider the sequence (8), and define the set $\Lambda$ as follows

$$
\Lambda=\left\{\lambda \mid \lambda=k_{s+1} / b_{s+1}, P_{s}(\lambda)=0, \text { for some } s=1, \ldots, n-1\right\} .
$$

If $\Lambda \cap(a, b)=\emptyset$, then the sequence (8) forms a Sturm sequence in the interval $(a, b)$.

Proof: From (8), the first condition in Definition 1 is satisfied. Consider the second condition. Let $\lambda^{*}$ be an arbitrarily selected element in $(a, b)$. Then by assumption $\lambda^{*} \notin \Lambda$, which implies that if $P_{i}\left(\lambda^{*}\right)=0$ for $i=1, \ldots, n-1$, then $k_{i+1}-\lambda^{*} b_{i+1} \neq 0$ and $P_{i+1}\left(\lambda^{*}\right) P_{i-1}\left(\lambda^{*}\right) \leq 0$ from (9). The equality sign cannot be achieved due to the following observation: if $P_{i+1}\left(\lambda^{*}\right) P_{i-1}\left(\lambda^{*}\right)=0$, then from $(9), P_{i+1}\left(\lambda^{*}\right)=P_{i-1}\left(\lambda^{*}\right)=0$. Since $P_{i-1}\left(\lambda^{*}\right)=0$ and $\lambda^{*} \notin \Lambda$ then $k_{i}-\lambda^{*} b_{i} \neq 0$ which means from (9) with $i$ replaced by $i-1$ that $P_{i-2}\left(\lambda^{*}\right)=0$. In this way, one has $P_{i-2}\left(\lambda^{*}\right)=P_{i-3}\left(\lambda^{*}\right)=\ldots=P_{0}\left(\lambda^{*}\right)=0$, which contradicts with $P_{0}\left(\lambda^{*}\right) \equiv 1$. Therefore, the second condition in Definition 1 is also satisfied. This establishes the proposition.

Evidently (8) is not always a Sturm sequence, as there may exist the situation where $k_{s+1} / b_{s+1}$ happens to be a zero of $P_{s}(\lambda)$. We will therefore need to derive the properties of the zeros of this sequence from first principles. We begin with the following Proposition.

Proposition 2. Consider the $n$-DOF mass-chain system shown in Fig. 1. Suppose $\lambda_{0} \in \Lambda$ defined in (10), namely there is an integer $s$ with $1 \leq s \leq n-1$, such that $b_{s+1}>0$, $\lambda_{0}=k_{s+1} / b_{s+1}$ and $P_{s}\left(\lambda_{0}\right)=0$. Then $P_{s+1}\left(\lambda_{0}\right)=P_{s+2}\left(\lambda_{0}\right)=\ldots=P_{n}\left(\lambda_{0}\right)=0$. In particular, all elements of $\Lambda$ are eigenvalues of the system.

Proof: From (9) with $i=s$, one has $P_{s+1}\left(\lambda_{0}\right)=0$. Replacing $i$ with $s+1, s+2, \ldots$, $n-1$ in (9), one obtains $P_{s+2}\left(\lambda_{0}\right)=0, P_{s+3}\left(\lambda_{0}\right)=0, \ldots, P_{n}\left(\lambda_{0}\right)=0$.

Based on Proposition 2, in what follows, we divide the discussion into two situations: one is for the eigenvalues not in $\Lambda$ and the other is for the eigenvalues in $\Lambda$. It will be proved that all the eigenvalues not in $\Lambda$ are simple, i.e., distinct, while eigenvalues in $\Lambda$ have the possibility to be multiple eigenvalues. 


\subsection{Eigenvalues not in $\Lambda$}

We first show that the zeros of $P_{r}(\lambda)$ which are not in $\Lambda$ are distinct from the zeros of $P_{r+1}(\lambda)$.

Proposition 3. Consider the sequence defined in (8). Suppose $\lambda_{0} \notin \Lambda$ and $P_{r}\left(\lambda_{0}\right)=0$ for some $r$ with $1 \leq r \leq n-1$. Then $P_{r+1}\left(\lambda_{0}\right) \neq 0$.

Proof: Suppose to the contrary that $P_{r+1}\left(\lambda_{0}\right)=0$. Then from (9) with $i=r$,

$$
\left(k_{r+1}-\lambda_{0} b_{r+1}\right)^{2} P_{r-1}\left(\lambda_{0}\right)=0,
$$

which implies $P_{r-1}\left(\lambda_{0}\right)=0$, since $P_{r}\left(\lambda_{0}\right)=0$ and $\lambda_{0} \notin \Lambda$. Using (9) successively in this way, one has $P_{r-1}\left(\lambda_{0}\right)=P_{r-2}\left(\lambda_{0}\right)=\ldots=P_{0}\left(\lambda_{0}\right)=0$, which contradicts with $P_{0}(\lambda) \equiv 1$. This establishes the proposition.

Lemma 1 (Separation theorem). [32, Page 340] Let $\mathbf{A}$ and $\mathbf{B}$ be symmetric matrices with $\mathbf{B}$ positive definite, and let $\mathbf{A}_{r}$ and $\mathbf{B}_{r}$ denote the leading principal submatrices of order $r$. Then the zeros of $\operatorname{det}\left(\mathbf{A}_{\mathbf{r}}-\lambda \mathbf{B}_{r}\right)$ separate those of $\operatorname{det}\left(\mathbf{A}_{\mathbf{r}+\mathbf{1}}-\lambda \mathbf{B}_{r+1}\right)$, namely,

$$
y_{1} \leq x_{1} \leq y_{2} \leq x_{2} \leq \ldots x_{r} \leq y_{r+1},
$$

where $x_{i}(i=1, \ldots, r)$ and $y_{i}(i=1, \ldots, r+1)$ are the zeros of the respective determinants.

Using Proposition 3 and Lemma 1 we derive the following theorem.

Theorem 1. Consider the sequence $P_{r}(\lambda)$ defined in (8) and the set $\Lambda$ defined in (10). The zeros of $P_{r}(\lambda), r=1, \ldots, n$, which are not in $\Lambda$ are simple.

Proof: From Lemma 1 , let $x_{i}(i=1, \ldots, r)$ be the zeros of $P_{r}(\lambda)$ and $y_{i}(i=1, \ldots, r+1)$ be the zeros of $P_{r+1}(\lambda)$ for $r=1, \ldots, n-1$, ordered as in (11). Suppose that $x_{k} \notin \Lambda$ for some $k$. Then from Proposition $3, P_{r+1}\left(x_{k}\right) \neq 0$, i.e.

$$
y_{k}<x_{k}<y_{k+1} .
$$

It follows that $x_{k}$ must be a simple zero of $P_{r}(\lambda)$. Similarly, if $\lambda_{0} \notin \Lambda$ is a non-simple zero of $P_{n}(\lambda)$, we must have

$$
y_{k}=x_{k}=y_{k+1}
$$

for some $k=1, \ldots, n-1$. Hence, $P_{n-1}\left(\lambda_{0}\right)=0$ contradicting Proposition 3 . This completes the proof.

From Theorem 1, it is straightforward to obtain the following corollaries.

Corollary 1. Consider the n-DOF mass-chain system shown in Fig. 1 and the set $\Lambda$ defined in (10). All the eigenvalues not in $\Lambda$ are simple. If the system has multiple eigenvalues, they must belong to $\Lambda$.

Corollary 2. If the set $\Lambda$ defined in (10) is an empty set, then all the eigenvalues of the system are simple.

Remark 1. For the conventional mass-chain system, that is the mass-chain system without inerters $\left(b_{1}=b_{2}=\ldots=b_{n}=0\right)$, the set $\Lambda$ is an empty set. This means that all eigenvalues of the mass-chain system without inerters are simple, which is a well-known result, e.g. [21, Page 52]. 


\subsection{Eigenvalues in $\Lambda$}

In Section 3.1, we have proved that all eigenvalues not in $\Lambda$ are simple, namely, if the system has multiple eigenvalues, they must belong to $\Lambda$. In this section, we will show that multiple eigenvalues are indeed possible.

Note that for each element in $\Lambda$, there may exist multiple indices corresponding to the element. We introduce the following definition of starting index.

Definition 1. Consider the sequence $P_{r}(\lambda), r=0,1, \ldots, n$ defined in (8) and the set $\Lambda$ defined in (10). For each element $\lambda_{0} \in \Lambda$, the smallest index $s$ such that $P_{s}\left(\lambda_{0}\right)=0$ and $\lambda_{0}=k_{s+1} / b_{s+1}$ is called the starting index for the element $\lambda_{0}$.

Proposition 4. Consider the sequence $P_{r}(\lambda), r=0,1, \ldots, n$ defined in (8) and the set $\Lambda$ defined in (10). Then the starting indices for different elements in $\Lambda$ are different. In other words, each element in $\Lambda$ has a unique starting index.

Proof: Denote $\lambda_{1}$ and $\lambda_{2}$ as two different elements in $\Lambda$, and assume the starting index for $\lambda_{1}$ and $\lambda_{2}$ is the same denoted as $s$. Then $k_{s+1} / b_{s+1}=\lambda_{1}$ and $k_{s+1} / b_{s+1}=\lambda_{2}$ should hold simultaneously, which contradicts with $\lambda_{1} \neq \lambda_{2}$.

As we will now show, it turns out that the sequence of multiplicities in $P_{r}(\lambda), r=$ $0,1, \ldots, n$ in $(8)$ is closely connected with the possibility of multiple eigenvalues. To facilitate the discussion we introduce the notation $\mu\left(P ; \lambda_{0}\right)$ to denote the multiplicity of a zero of the polynomial $P(\lambda)$ at $\lambda=\lambda_{0}$. We also define:

$$
\mu\left(P_{1}, P_{2}, \ldots, P_{r} ; \lambda_{0}\right)=\left[\mu\left(P_{1} ; \lambda_{0}\right), \mu\left(P_{2} ; \lambda_{0}\right), \ldots, \mu\left(P_{r} ; \lambda_{0}\right)\right] .
$$

Proposition 5. Consider the sequence $P_{r}(\lambda), r=0,1, \ldots, n$ in (8) and consider any $\lambda_{0}>0$. Then the difference in the zero multiplicities at $\lambda_{0}$ between adjacent terms in the sequence is no larger than 1 , namely $\left|\mu\left(P_{r} ; \lambda_{0}\right)-\mu\left(P_{r+1} ; \lambda_{0}\right)\right| \leq 1$ for $0 \leq r \leq n-1$.

Proof: For $\lambda_{0} \notin \Lambda$ this is a consequence of Theorem 1. Otherwise it follows directly from Lemma 1. Suppose $\lambda_{0}$ is a zero of multiplicity $m>1$ of $P_{r}(\lambda)$, where $r<n$. Let the zeros of $P_{r}(\lambda)$ be denoted by $x_{i}(i=1, \ldots, r)$ and those of $P_{r+1}(\lambda)$ by $y_{i}(i=1, \ldots, r+1)$. Then there is a $j \geq 1$ such that

$$
\lambda_{0}=x_{j}=\ldots=x_{j+m-1}
$$

From Lemma 1,

$$
x_{j}=y_{j+1}=x_{j+1}=\ldots=y_{j+m-1}=x_{j+m-1},
$$

and hence $\lambda_{0}$ is a zero of multiplicity at least $m-1$ of $P_{r+1}(\lambda)$. Similarly, if $m \geq 1, \lambda_{0}$ cannot be a zero of $P_{r+1}(\lambda)$ of multiplicity greater than $m+1$.

Proposition 6. Consider the sequence $P_{r}(\lambda)$ defined in (8) and the set $\Lambda$ defined in (10). Let $\lambda_{0} \in \Lambda$ with starting index s. Then

1. $\mu\left(P_{s} ; \lambda_{0}\right)=1$ and $\mu\left(P_{s-1} ; \lambda_{0}\right)=0$;

2. if $\mu\left(P_{s+1} ; \lambda_{0}\right)=2$ and $s \leq n-2$ then $\mu\left(P_{s+2} ; \lambda_{0}\right)=1$. 
Proof: 1) If $\lambda_{0}$ belongs to $\Lambda$ with a starting index $s$, then $P_{s}\left(\lambda_{0}\right)=0$ holds according to the definition of starting index. If $P_{s-1}\left(\lambda_{0}\right)=0$, then $k_{s}-\lambda_{0} b_{s} \neq 0$ as otherwise $(s-1)$ should be the starting index for $\lambda_{0}$. If $P_{s-1}\left(\lambda_{0}\right)=0$ and $k_{s}-\lambda_{0} b_{s} \neq 0$, from (9) with $i=s-1$, one has $P_{s-2}\left(\lambda_{0}\right)=0$. Similarly, since $k_{s-1}-\lambda_{0} b_{s-1} \neq 0$, then $P_{s-3}\left(\lambda_{0}\right)=0$. Repeating this procedure, one has $P_{0}\left(\lambda_{0}\right)=0$, contradicting with $P_{0}(\lambda) \equiv 1$. Therefore $P_{s-1}\left(\lambda_{0}\right) \neq 0$. It then follows from Proposition 5 that $\mu\left(P_{s} ; \lambda_{0}\right)=1$.

2) From (9) with $i=s$ we see that

$$
\lambda_{0}=\frac{k_{s+1}+k_{s+2}}{m_{s+1}+b_{s+1}+b_{s+2}}=\frac{k_{s+2}}{m_{s+1}+b_{s+2}} .
$$

If $\mu\left(P_{s+1} ; \lambda_{0}\right)=2$, then from (9) with $i=s+1$ we see that $\lambda_{0}=k_{s+2} / b_{s+2}$ which contradicts (12).

Proposition 7. Consider the sequence $P_{r}(\lambda), r=0,1, \ldots, n$ defined in (8) and the set $\Lambda$ defined in (10) for $n \geq 2$. Let $\lambda_{0} \in \Lambda$ be such that $\mu\left(P_{n} ; \lambda_{0}\right)=m \geq 2$. If $\mu\left(P_{n-1} ; \lambda_{0}\right)=m-1$ then $\mu\left(P_{n-2} ; \lambda_{0}\right) \geq m-1$.

Proof: Suppose $\mu\left(P_{n-2} ; \lambda_{0}\right)=m-2$. Equation (9) with $i=n-1$ takes the form

$$
P_{n}(\lambda)=\left(k_{n}-\lambda\left(m_{n}+b_{n}\right)\right) P_{n-1}(\lambda)-\left(k_{n}-\lambda b_{n}\right)^{2} P_{n-2}(\lambda) .
$$

Dividing (13) by $\left(\lambda-\lambda_{0}\right)^{m-2}$ and setting $\lambda=\lambda_{0}$ implies $\lambda_{0}=k_{n} / b_{n}$. Then from (13), $\mu\left(P_{n} ; \lambda_{0}\right)=m$ implies $\lambda_{0}=k_{n} /\left(m_{n}+b_{n}\right)$, which is a contradiction.

Corollary 3. For the mass-chain system shown in Fig. 1 with $n \geq 2$ it is impossible to assign an eigenvalue of multiplicity $n$.

Proof: Proposition 5 implies $\mu\left(P_{1}, P_{2}, \ldots, P_{n} ; \lambda_{0}\right)=[1,2, \ldots, n]$ which is a contradiction to Proposition 7.

Proposition 8. Consider the mass-chain system shown in Fig. 1. For $n<3$ it is not possible for $\lambda_{0}>0$ to be a double eigenvalue (i.e. $\mu\left(P_{n} ; \lambda_{0}\right)=2$ ). For $n=3$ a necessary and sufficient condition for $\lambda_{0}>0$ to be a double eigenvalue (i.e. $\left.\mu\left(P_{3} ; \lambda_{0}\right)=2\right)$ is

$$
\lambda_{0}=\frac{k_{1}}{m_{1}+b_{1}}=\frac{k_{2}}{b_{2}}=\frac{k_{3}}{m_{2} \wedge m_{3}+b_{3}} .
$$

where $m_{2} \wedge m_{3}$ denotes $m_{2} m_{3} /\left(m_{2}+m_{3}\right)$.

Proof: Corollary 3 shows the impossibility for $n=2$. For $n=3$ Propositions 6 and 7 show that a double eigenvalue can be obtained only if

$$
\mu\left(P_{1}, P_{2}, P_{3} ; \lambda_{0}\right)=[1,1,2] .
$$

$P_{1}\left(\lambda_{0}\right)=0$ implies $\lambda_{0}=\left(k_{1}+k_{2}\right) /\left(m_{1}+b_{1}+b_{2}\right) . P_{2}\left(\lambda_{0}\right)=0$ and (9) with $i=1$ show that $\lambda_{0}=k_{2} / b_{2}$ which gives the first two equalities in (14). Substituting for $P_{2}$ from (9) with $i=1$ (while omitting the $P_{0}$ term which has a double zero at $\lambda_{0}$ ) into the expression for $P_{3}$ from (9) with $i=2$ shows that

$$
\left[\left(k_{3}-\lambda\left(m_{3}+b_{3}\right)\right)\left(k_{2}+k_{3}-\lambda\left(m_{2}+b_{2}+b_{3}\right)\right)-\left(k_{3}-\lambda b_{3}\right)^{2}\right] P_{1}(\lambda)
$$


must have a double zero at $\lambda_{0}$. This means (since $P_{1}$ may only have a single zero at $\lambda_{0}$ ) that

$$
0=\left(k_{3}-\lambda_{0}\left(m_{3}+b_{3}\right)\right)\left(k_{3}-\lambda_{0}\left(m_{2}+b_{3}\right)\right)-\left(k_{3}-\lambda_{0} b_{3}\right)^{2}
$$

which gives the third equality in (14).

Remark 2. It can be seen that elements of $\Lambda$ do not need to be multiple eigenvalues. For example, if $n=3, \lambda_{0}=k_{1} /\left(m_{1}+b_{1}\right)=k_{2} / b_{2} \neq k_{3} /\left(m_{2} \wedge m_{3}+b_{3}\right)$ then $\mu\left(P_{1}, P_{2}, P_{3} ; \lambda_{0}\right)=$ $[1,1,1]$ and $\lambda_{0}$ is simple.

Proposition 9. Consider the sequence $P_{r}(\lambda), r=0,1, \ldots, n$ defined in (8) and the set $\Lambda$ defined in (10). Suppose $n \geq 3$ and

$$
\mu\left(P_{r}, P_{r+1}, P_{r+2} ; \lambda_{0}\right)=[m-1, m, m+1]
$$

with $m \geq 1$ and $r \leq n-3$. Then:

1. $\mu\left(P_{r+3} ; \lambda_{0}\right)=m$;

2. if $r+4 \leq n$ then $\mu\left(P_{r+4} ; \lambda_{0}\right) \geq m$.

Proof: 1) Taking equation (9) with $i=r+1$, dividing by $\left(\lambda-\lambda_{0}\right)^{m-1}$ and setting $\lambda=\lambda_{0}$ implies $\lambda_{0}=k_{r+2} / b_{r+2}$. In order that $\mu\left(P_{r+2} ; \lambda_{0}\right)=m+1$, from (9) with $i=r+1$, we now need

$$
\lambda_{0}=\frac{k_{r+2}+k_{r+3}}{m_{r+2}+b_{r+2}+b_{r+3}}=\frac{k_{r+3}}{m_{r+2}+b_{r+3}} .
$$

Now taking equation (9) with $i=r+2$ and dividing by $\left(\lambda-\lambda_{0}\right)^{m}$ we see that $P_{r+3} /\left(\lambda-\lambda_{0}\right)^{m}$ is finite and non-zero at $\lambda=\lambda_{0}$, since $\lambda_{0} \neq k_{r+3} / b_{r+3}$ from (16). Hence $\mu\left(P_{r+3} ; \lambda_{0}\right)=m$.

2) The result follows directly from (9) with $i=r+3$.

Proposition 10. Consider the mass-chain system shown in Fig. 1. For $n<5$ it is not possible for $\lambda_{0}>0$ to be a triple eigenvalue (i.e. $\mu\left(P_{n} ; \lambda_{0}\right)=3$ ). For $n=5$ a necessary and sufficient condition for $\lambda_{0}>0$ to be a triple eigenvalue (i.e. $\mu\left(P_{5} ; \lambda_{0}\right)=3$ ) is

$$
\lambda_{0}=\frac{k_{1}}{m_{1}+b_{1}}=\frac{k_{2}}{b_{2}}=\frac{k_{3}}{m_{2} \wedge m_{3}+b_{3}}=\frac{k_{4}}{b_{4}}=\frac{k_{5}}{m_{4} \wedge m_{5}+b_{5}} .
$$

Proof: Corollary 3 shows the impossibility for $n=3$. For $n=4$ Propositions 6 and 7 show that a triple eigenvalue is impossible. For $n=5$ Propositions 6,7 and 9 show that a triple eigenvalue can be obtained only if

$$
\mu\left(P_{1}, P_{2}, \ldots, P_{5} ; \lambda_{0}\right)=[1,1,2,2,3] .
$$

The first two equalities in (17) follow as in the proof of Proposition 8. In order that

$$
\mu\left(P_{2}, P_{3}, P_{4} ; \lambda_{0}\right)=[1,2,2]
$$

from (9) with $i=3$ we see that $\lambda_{0}=k_{4} / b_{4}$. As in the proof of Proposition 8 the expression for $P_{3}$ from (9) with $i=2$ implies that

$$
\left[\left(k_{3}+k_{4}-\lambda\left(m_{3}+b_{3}+b_{4}\right)\right)\left(k_{2}+k_{3}-\lambda\left(m_{2}+b_{2}+b_{3}\right)\right)-\left(k_{3}-\lambda b_{3}\right)^{2}\right] P_{1}(\lambda)
$$


must have a double zero at $\lambda_{0}$, from which (15) follows, which gives the third equality in (17). Similarly the expression for $P_{5}$ from (9) with $i=4$ implies that

$$
\left[\left(k_{5}-\lambda\left(m_{5}+b_{5}\right)\right)\left(k_{4}+k_{5}-\lambda\left(m_{4}+b_{4}+b_{5}\right)\right)-\left(k_{5}-\lambda b_{5}\right)^{2}\right] P_{3}(\lambda)
$$

must have a triple zero at $\lambda_{0}$, from which the fifth equality in (17) follows.

Theorem 2. Consider the mass-chain system shown in Fig. 1. For $n<2 m-1$ it is not possible for $\lambda_{0}>0$ to be an eigenvalue of multiplicity $m>1$ (i.e. $\left.\mu\left(P_{n} ; \lambda_{0}\right)=m\right)$. For $n=2 m-1$ a necessary and sufficient condition for $\mu\left(P_{n} ; \lambda_{0}\right)=m$ is that the following equalities hold:

$$
\begin{aligned}
& \lambda_{0}=\frac{k_{1}}{m_{1}+b_{1}}=\frac{k_{3}}{m_{2} \wedge m_{3}+b_{3}}=\frac{k_{5}}{m_{4} \wedge m_{5}+b_{5}}=\cdots=\frac{k_{n}}{m_{n-1} \wedge m_{n}+b_{n}}, \\
& \lambda_{0}=\frac{k_{2}}{b_{2}}=\frac{k_{4}}{b_{4}}=\cdots=\frac{k_{n-1}}{b_{n-1}} .
\end{aligned}
$$

Proof: Let $\mu\left(P_{n} ; \lambda_{0}\right)=m$. Propositions 5 and 7 imply $\mu\left(P_{n-1} ; \lambda_{0}\right) \geq m-1$ and $\mu\left(P_{n-2} ; \lambda_{0}\right) \geq m-1$. Propositions 5 and 9 then imply $\mu\left(P_{n-3} ; \lambda_{0}\right) \geq m-2$ and $\mu\left(P_{n-4} ; \lambda_{0}\right) \geq$ $m-2$. In the same way, Propositions 5 and 9 then imply $\mu\left(P_{n-5} ; \lambda_{0}\right) \geq m-3$ and $\mu\left(P_{n-6} ; \lambda_{0}\right) \geq m-3$. Continuing in this way $\mu\left(P_{n-2 m+3} ; \lambda_{0}\right) \geq 1$ which means that $n-$ $2 m+3 \geq 1$. Now if $n-2 m+3=1$ there is a contradiction to Proposition 6 , therefore $n-2 m+3>1$ which means $n>2 m-2$. This proves the first part of the proposition.

In order for $\mu\left(P_{n} ; \lambda_{0}\right)=m$ with $n=2 m-1$ we must therefore have:

$$
\mu\left(P_{1}, P_{2}, \ldots, P_{n} ; \lambda_{0}\right)=[1,1,2,2, \ldots, m-1, m-1, m] .
$$

The first equality in (18) and (19) follow as in the proof of Proposition 10. In order that

$$
\mu\left(P_{2}, P_{3}, P_{4} ; \lambda_{0}\right)=[1,2,2]
$$

from (9) with $i=3$ we see that $\lambda_{0}=k_{4} / b_{4}$. In order that

$$
\mu\left(P_{4}, P_{5}, P_{6} ; \lambda_{0}\right)=[2,3,3]
$$

from (9) with $i=5$ we see that $\lambda_{0}=k_{6} / b_{6}$. Continuing in this way we obtain (19).

As in the proof of Proposition 10 the expression for $P_{3}$ from (9) with $i=2$ implies that

$$
\left[\left(k_{3}+k_{4}-\lambda\left(m_{3}+b_{3}+b_{4}\right)\right)\left(k_{2}+k_{3}-\lambda\left(m_{2}+b_{2}+b_{3}\right)\right)-\left(k_{3}-\lambda b_{3}\right)^{2}\right] P_{1}(\lambda)
$$

must have a double zero at $\lambda_{0}$, which gives the second equality in (18). Similarly the expression for $P_{5}$ from (9) with $i=4$ implies that

$$
\left[\left(k_{5}+k_{6}-\lambda\left(m_{5}+b_{5}+b_{6}\right)\right)\left(k_{4}+k_{5}-\lambda\left(m_{4}+b_{4}+b_{5}\right)\right)-\left(k_{5}-\lambda b_{5}\right)^{2}\right] P_{3}(\lambda)
$$

must have a triple zero at $\lambda_{0}$, which gives the third equality in (18). Continuing in this way we obtain (18). 


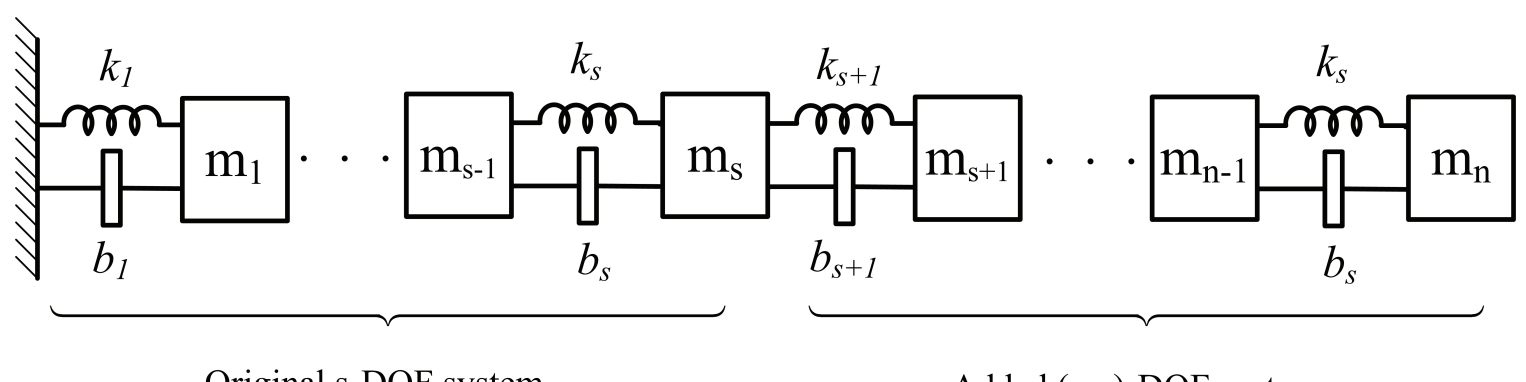

Original s-DOF system

Added (n-s)-DOF system

Figure 2: Augment an $s$-DOF system to an $n$-DOF system.

\subsection{Structural modification}

The results in the previous sections can also be used to the problem of structure modification while preserving the natural frequency of the original system as follows.

Problem 2. As shown in Fig. 2, augment the s-DOF system to an n-DOF system where $n>s$. Let $\lambda_{0}$ be a natural frequency of the $s$-DOF system. Determine the augmented inertances and spring stiffnesses such that $\lambda_{0}$ remains to be a natural frequency of the $n$ DOF system.

Problem 2 can easily be solved by choosing $k_{s+1} / b_{s+1}=\lambda_{0}$. This can be demonstrated as follows. Denote the sequence (8) for the original $s$-DOF system as $P_{0}(\lambda), P_{1}(\lambda), \ldots, P_{s}^{\prime}(\lambda)$, and denote the sequence (8) for the augmented $n$-DOF system as $P_{0}(\lambda), P_{1}(\lambda), \ldots, P_{s}(\lambda)$, $P_{s+1}(\lambda), \ldots, P_{n}(\lambda)$. Then, one has

$$
\begin{aligned}
& P_{s}^{\prime}(\lambda)=\left(k_{s}-\lambda\left(m_{s}+b_{s}\right)\right) P_{s-1}(\lambda)-\left(k_{s}-\lambda b_{s}\right)^{2} P_{s-2}(\lambda) \\
& P_{s}(\lambda)=\left(k_{s}+k_{s+1}-\lambda\left(m_{s}+b_{s}+b_{s+1}\right)\right) P_{s-1}(\lambda)-\left(k_{s}-\lambda b_{s}\right)^{2} P_{s-2}(\lambda) .
\end{aligned}
$$

Assume $\lambda_{0}=k_{s+1} / b_{s+1}$. It is straightforward that if $P_{s}^{\prime}\left(\lambda_{0}\right)=0$ then $P_{s}\left(\lambda_{0}\right)=0$. From Proposition $2, \lambda_{0}$ is also a zero of $P_{s+1}(\lambda), P_{s+2}(\lambda), \ldots, P_{n}(\lambda)$.

\section{Placement of distinct eigenvalues}

In Section 3, it has been proved that it is possible for mass-chain systems with inerters shown in Fig. 1 to have multiple eigenvalues, which is quite different from those without inerters whose eigenvalues are always simple. It has also been proved that if the given numbers are not strictly distinct, arbitrarily given numbers cannot always be assigned as the eigenvalues of the system by using only inerters and springs. In this section, the case where all the given numbers are distinct will be studied, and it will be proved that if the arbitrarily given numbers are distinct, they can always be assigned as the natural frequencies of mass-chain systems by using inerters and springs.

Specifically, the problem studied in this section is:

Problem 3. Let $m_{i}>0, i=1, \ldots, n$, be given and fixed, is it possible to use only inerters and springs placed between adjacent masses as shown in Fig. 1 to realize any arbitrarily given numbers $0<\lambda_{1}<\lambda_{2}<\ldots<\lambda_{n}$ as the eigenvalues of the mass-chain system? If so, what is the minimal number of inerters required to achieve this? 


\subsection{Two-degree-of-freedom system}

Before addressing Problem 3, we first show that if the masses $m_{i}>0, i=1, \ldots, n$, are given and fixed, it is not always possible to realize any arbitrarily given numbers $0<\lambda_{1}<$ $\lambda_{2}<\ldots<\lambda_{n}$ as the eigenvalues of the mass-chain system by using springs only. In other words, inerters are necessary if the masses are fixed. The following result shows that for a 2-DOF mass-chain system the eigenvalues must be sufficiently separated.

Theorem 3. Consider the 2-DOF mass-chain system without inerters. Let $m_{1}$ and $m_{2}$ be given and fixed. The values $\lambda_{1}>0$ and $\lambda_{2}>0$ may be assigned as the eigenvalues of the system by choice of the springs $k_{1}$ and $k_{2}$ if and only if

$$
\left(\sqrt{\frac{\lambda_{1}}{\lambda_{2}}}-\sqrt{\frac{\lambda_{2}}{\lambda_{1}}}\right)^{2} \geq 4 \alpha
$$

where $\alpha=m_{2} / m_{1}$ is the mass ratio.

Proof: The $\mathbf{M}$ and $\mathbf{K}$ matrix for the 2-DOF system without inerters are

$$
\mathbf{M}=\left[\begin{array}{ll}
m_{1} & \\
& m_{2}
\end{array}\right], \mathbf{K}=\left[\begin{array}{cc}
k_{1}+k_{2} & -k_{2} \\
-k_{2} & k_{2}
\end{array}\right] .
$$

The characteristic equation is

$$
\Delta(\lambda)=m_{1} m_{2} \lambda^{2}-\left(k_{2} m_{1}+k_{1} m_{2}+k_{2} m_{2}\right) \lambda+k_{1} k_{2}=0 .
$$

Denote $\rho_{1}=k_{1} / m_{1}, \rho_{2}=k_{2} / m_{2}, \alpha=m_{2} / m_{1}$. Then

$$
\Delta(\lambda)=\lambda^{2}-\left(\rho_{1}+\rho_{2}+\rho_{2} \alpha\right) \lambda+\rho_{1} \rho_{2}=0 .
$$

Since $m_{1}$ and $m_{2}$ are given, $k_{1}$ and $k_{2}$ are free, then $\rho_{1}$ and $\rho_{2}$ are free, but $\alpha$ is fixed. Therefore, the problem is equivalent to: given $\lambda_{1}>0, \lambda_{2}>0$, and given $\alpha>0$, whether there always exist $\rho_{1}>0, \rho_{2}>0$, such that $\lambda_{1}$ and $\lambda_{2}$ are the solutions of (23)?

Suppose that $\lambda_{1}>0$ and $\lambda_{2}>0$ are the solutions of equation (23), then

$$
\begin{aligned}
\lambda_{1}+\lambda_{2} & =\rho_{1}+(1+\alpha) \rho_{2}, \\
\lambda_{1} \lambda_{2} & =\rho_{1} \rho_{2} .
\end{aligned}
$$

Substituting $\rho_{1}$ from (24) into (25), one obtains

$$
(1+\alpha) \rho_{2}^{2}-\rho_{2}\left(\lambda_{1}+\lambda_{2}\right)+\lambda_{1} \lambda_{2}=0
$$

and $\rho_{1}=\lambda_{1} \lambda_{2} / \rho_{2}$. Therefore a necessary and sufficient condition for $\lambda_{1}>0$ and $\lambda_{2}>0$ to be the solutions of Equation (23) is that (26) has a positive solution. This is equivalent to the discriminant of (26) being non-negative, i.e. $\left(\lambda_{1}+\lambda_{2}\right)^{2}-4(1+\alpha) \lambda_{1} \lambda_{2} \geq 0$ which yields $(22)$. 


\subsection{Necessary and sufficient condition}

In Section 4.1, it has been demonstrated that the realizable set of the eigenvalues for mass-chain systems without inerters is restricted. However, in this section, we will show that such a restriction can be removed by using at most $(n-1)$ inerters for an $n$-DOF mass-chain system.

Theorem 4. Consider the $n$-DOF mass-chain system with inerters, where the masses $m_{i}>$ $0, i=1, \ldots, n$, are given and fixed. Let $0<\lambda_{1}<\lambda_{2}<\ldots<\lambda_{n}$ be arbitrarily given numbers. Then $\lambda_{i}, i=1, \ldots, n$, may be assigned as the eigenvalues of the $n$-DOF system using $n$ springs and $(n-1)$ inerters but not necessarily using $n$ springs and $(n-2)$ inerters.

The proof of Theorem 4 is based on a multiplicative inverse eigenvalue problem (MIEP) formulation. The MIEP is stated as follows.

Problem 4 (MIEP [30]). Given a Hermitian matrix $\mathbf{A} \in \mathbf{R}^{n \times n}$ and $n$ real positive distinct numbers $s_{i}, i=1, \ldots, n$, find $n$ real positive numbers $d_{i}, i=1, \ldots, n$, such that the eigenvalues of the matrix $\mathbf{D A}$ are $s_{i}, i=1, \ldots, n$, where $\mathbf{D}=\operatorname{diag}\left\{d_{1}, d_{2}, \ldots, d_{n}\right\}$.

The following two Lemmas provide the basis for sufficient and necessary conditions for the solvability of the MIEP, which will be employed in the proof of Theorem 4.

Lemma 1. Let $\mathbf{A}=\left[a_{i j}\right]$ be an $n \times n$ real matrix. Assume that $a_{i i}=1, i=1, \ldots, n$, $\max _{i} P_{i} \leq 1 / 2$ and no two of the intervals $\left[s_{i}\left(1-2 P_{i}\right), s_{i}\left(1+P_{i}\right) /\left(1-P_{i}\right)\right], i=1, \ldots, n$ intersect, where the $s_{i}$ are real positive distinct numbers, and

$$
P_{i}=\sum_{j=1, j \neq i}^{n}\left|a_{i j}\right| .
$$

Then there exists a diagonal matrix $\mathbf{D}$ with positive diagonal entries such that $\mathbf{D A}$ has eigenvalues $s_{i}, i=1, \ldots, n$.

Proof: This lemma is a restriction of Theorem 2.1 in [33] to the case that the $s_{i}$ are positive. It can be verified from the proof in [33, Theorem 2.1] that the diagonal entries of $\mathbf{D}$ are positive real numbers in such a case.

Lemma 2. [34] Let $\mathbf{A}=\left[a_{i j}\right]$ be an $n \times n$ Hermitian matrix with $a_{i i}=1$ for $i=1, \ldots, n$. A necessary condition for the existence of a positive diagonal matrix $\mathbf{D}=\operatorname{diag}\left\{d_{1}, d_{2}, \ldots, d_{n}\right\}$ such that the eigenvalues of the matrix $\mathbf{D A}$ are $s_{i}$ where $s_{1} \geq s_{2} \geq \ldots \geq s_{n}>0$ is

$$
\sum_{1 \leq i<j \leq n}\left(s_{i}-s_{j}\right)^{2} \geq s_{n}^{2} n\left\|\mathbf{A}^{(0)}\right\|_{F}^{2}
$$

where $\mathbf{A}^{(0)}=\mathbf{A}-\operatorname{diag}\left\{a_{11}, \ldots, a_{n n}\right\}$ and $\|\cdot\|_{F}$ denotes the Frobenius norm.

Proof of Theorem 4: The underlying idea of the proof of Theorem 4 is: first, formulate the problem as an MIEP shown in Problem 4; then, prove the sufficiency by demonstrating that the sufficient conditions in Lemma 1 are satisfied if $(n-1)$ inerters and $n$ springs are employed; finally, prove the necessity by showing that the necessary conditions in Lemma 2 are not always satisfied if only $(n-2)$ inerters and $n$ springs are employed. 
Consider the first step, that is, to formulate the problem as an MIEP. Note that the matrix $\mathbf{K}$ and matrix $\mathbf{B}$ in (4) and (3), respectively, have the same structure, which can be rewritten as $[21]$

$$
\begin{aligned}
& \mathbf{K}=\mathbf{E} \hat{\mathbf{K}} \mathbf{E}^{T} \\
& \mathbf{B}=\mathbf{E} \hat{\mathbf{B}} \mathbf{E}^{T}
\end{aligned}
$$

where $\hat{\mathbf{K}}=\operatorname{diag}\left\{k_{1}, k_{2}, \ldots, k_{n}\right\}, \hat{\mathbf{B}}=\operatorname{diag}\left\{b_{1}, b_{2}, \ldots, b_{n}\right\}$,

$$
\mathbf{E}=\left[\begin{array}{ccccc}
1 & -1 & & & \\
& 1 & -1 & & \\
& & \ddots & \ddots & \\
& & & 1 & -1 \\
& & & & 1
\end{array}\right], \mathbf{E}^{-1}=\left[\begin{array}{ccccc}
1 & 1 & 1 & \ldots & 1 \\
& 1 & 1 & \ldots & 1 \\
& & \ddots & \ddots & \vdots \\
& & & 1 & 1 \\
& & & & 1
\end{array}\right]
$$

Since $\mathbf{E}$ is nonsingular (5) is equivalent to

$$
\operatorname{det}\left(\hat{\mathbf{K}}-\lambda\left(\hat{\mathbf{M}}_{0}+\hat{\mathbf{B}}\right)\right)=0
$$

where

$$
\hat{\mathbf{M}}_{0}=\mathbf{E}^{-1} \mathbf{M}_{\mathbf{0}} \mathbf{E}^{-T}=\left[\begin{array}{ccccc}
\hat{m}_{1} & \hat{m}_{2} & \hat{m}_{3} & \ldots & \hat{m}_{n} \\
\hat{m}_{2} & \hat{m}_{2} & \hat{m}_{3} & \ldots & \hat{m}_{n} \\
\hat{m}_{3} & \hat{m}_{3} & \hat{m}_{3} & \ldots & \hat{m}_{n} \\
\vdots & \vdots & \vdots & \ddots & \vdots \\
\hat{m}_{n} & \hat{m}_{n} & \hat{m}_{n} & \ldots & \hat{m}_{n}
\end{array}\right]
$$

and $\hat{m}_{i}=\sum_{j=i}^{n} m_{j}, i=1, \ldots, n$. It is clear that $\hat{m}_{1}>\hat{m}_{2}>\ldots>\hat{m}_{n}=m_{n}$.

Denoting $\hat{\mathbf{M}}=\hat{\mathbf{M}}_{0}+\hat{\mathbf{B}}$, we can decompose $\hat{\mathbf{M}}$ as

$$
\hat{\mathbf{M}}=\widetilde{\mathbf{M}}_{0} \widetilde{\mathbf{M}} \widetilde{\mathbf{M}}_{0}
$$

where $\widetilde{\mathbf{M}}_{0}=\operatorname{diag}\left\{\sqrt{\hat{m}_{1}+b_{1}}, \sqrt{\hat{m}_{2}+b_{2}}, \ldots, \sqrt{\hat{m}_{n}+b_{n}}\right\}$, and $\widetilde{\mathbf{M}}=\left[\widetilde{m}_{i j}\right]$ with

$$
\widetilde{m}_{i j}=\left\{\begin{array}{cl}
1, & j=i, \\
\widetilde{m}_{j i}=\frac{\hat{m}_{j}}{\sqrt{\left(\hat{m}_{i}+b_{i}\right)\left(\hat{m}_{j}+b_{j}\right)}}, & j>i .
\end{array}\right.
$$

Similarly, since $\widetilde{\mathbf{M}}_{0}$ is nonsingular, (30) is equivalent to

$$
\operatorname{det}(\widetilde{\mathbf{K}}-\lambda \widetilde{\mathbf{M}})=0
$$

where $\widetilde{\mathbf{K}}=\widetilde{\mathbf{M}}_{0}^{-1} \hat{\mathbf{K}} \widetilde{\mathbf{M}}_{0}^{-1}=\operatorname{diag}\left\{k_{1} /\left(\hat{m}_{1}+b_{1}\right), k_{2} /\left(\hat{m}_{2}+b_{2}\right), \ldots, k_{n} /\left(\hat{m}_{n}+b_{n}\right)\right\}$. Furthermore, since $\widetilde{\mathbf{K}}$ is nonsingular, (31) is equivalent to

$$
\operatorname{det}\left(\mathbf{I}-\widetilde{\mathbf{K}}^{-1} \widetilde{\mathbf{M}} \lambda\right)=0
$$


Suppose $0<\lambda_{1}<\lambda_{2}<\ldots<\lambda_{n}$ are arbitrarily given eigenvalues of the matrix pair $(\mathbf{K}, \mathbf{M})$ in (5), which are also the roots of (32). Then $\mu_{1}>\mu_{2}>\ldots>\mu_{n}>0$ with $\mu_{i}=1 / \lambda_{i}, i=1, \ldots, n$ are the eigenvalues of $\widetilde{\mathbf{K}}^{-1} \widetilde{\mathbf{M}}$. Therefore, the problem of investigating the eigenvalues of the matrix pair $(\mathbf{K}, \mathbf{M})$ is equivalent to the problem of investigating the eigenvalues of $\widetilde{\mathbf{K}}^{-1} \widetilde{\mathbf{M}}$.

Hence, the problem is to demonstrate that for arbitrarily given numbers $\mu_{1}>\mu_{2}>\ldots>$ $\mu_{n}>0$, it is sufficient and necessary to use $(n-1)$ inerters and $n$ springs such that $\mu_{i}$, $i=1, \ldots, n$, are the eigenvalues of $\widetilde{\mathbf{K}}^{-1} \widetilde{\mathbf{M}}$ for all possible values of masses. This problem is indeed an MIEP, if we view the matrices $\widetilde{\mathbf{K}}^{-1}$ and $\widetilde{\mathbf{M}}$ as the matrices $\mathbf{D}$ and $\mathbf{A}$ in Problem 4 , respectively.

Sufficiency: we will show that by carefully adjusting at most $(n-1)$ inertances, the sufficient conditions in Lemma 1 can always be satisfied, and hence, the existence of $n$ real positive spring stiffnesses can always be guaranteed.

To be consistent with the notations of Problem 4, we denote $\widetilde{\mathbf{K}}^{-1}$ and $\widetilde{\mathbf{M}}$ as $\mathbf{D}$ and $\mathbf{A}$, respectively. Also, by denoting

$$
\delta_{i}=\frac{b_{i}}{\hat{m}_{i}}, \rho_{i}=\frac{k_{i}}{\hat{m}_{i}}, i=1, \ldots, n,
$$

and $\alpha_{i}=\frac{\hat{m}_{i+1}}{\hat{m}_{i}}, i=1, \ldots, n-1$, we obtain

$$
\begin{aligned}
& \mathbf{A}=\widetilde{\mathbf{M}}=\left[a_{i j}\right] \\
& \mathbf{D}=\widetilde{\mathbf{K}}^{-1}=\operatorname{diag}\left\{d_{1}, d_{2}, \ldots, d_{n}\right\}
\end{aligned}
$$

where

$$
a_{i j}=\left\{\begin{array}{cl}
1, & j=i \\
a_{j i}=\frac{\sqrt{\alpha_{i} \alpha_{i+1} \ldots \alpha_{j-1}}}{\sqrt{\left(1+\delta_{i}\right)\left(1+\delta_{j}\right)}}, & j>i
\end{array},\right.
$$

and $d_{i}=\left(1+\delta_{i}\right) / \rho_{i}, i=1, \ldots, n$.

We now apply Lemma 1 . We note that the intervals $\left[\mu_{i}\left(1-2 P_{i}\right), \mu_{i}\left(1+P_{i}\right) /\left(1-P_{i}\right)\right]$, $i=1, \ldots, n$, each contain $\mu_{i}$ and have vanishing width as $\max _{i} P_{i} \rightarrow 0$. In particular, none of the intervals intersect if

$$
\frac{\lambda_{i+1}}{\lambda_{i}}=\frac{\mu_{i}}{\mu_{i+1}}>\frac{1+P_{i+1}}{\left(1-P_{i+1}\right)\left(1-2 P_{i}\right)}
$$

for $i=1, \ldots, n-1$. This may always achieved by making $\max _{i} P_{i}$ sufficiently small since the RHS of (36) may be made as close to 1 as desired, whereas the LHS is always greater than 1 , for each $i$. From (35), we can see that we may choose $\delta_{i}=0$ for an arbitrarily chosen $i$ in $1, \ldots, n$, and ensure that $\max _{i} P_{i}$ is as small as desired by choosing $\delta_{j}$ sufficiently large for $j=1, \ldots, n$ and $j \neq i$. This demonstrates that $n-1$ inerters are sufficient to satisfy the conditions in Lemma 1 . Then, Lemma 1 implies that there always exist positive $d_{i}$, $i=1, \ldots, n$, such that $\operatorname{diag}\left\{d_{1}, d_{2}, \ldots, d_{n}\right\} \mathbf{A}$ has eigenvalues $s_{i}, i=1, \ldots, n$. This means that we can always choose $\rho_{i}=\left(1+\delta_{i}\right) / d_{i}, i=1, \ldots, n$ to complete the assignment of the eigenvalues. This completes the proof of sufficiency.

Necessity: we will show that if only $(n-2)$ inerters and $n$ springs are employed, the necessary conditions in Lemma 2 are not satisfied for all $\mu_{1}>\mu_{2}>\ldots>\mu_{n}>0$. 
Suppose that only $(n-2)$ inerters are employed. Then there exist $i$ and $j$ with $i \neq j$ such that $\delta_{i}=\delta_{j}=0$. Without loss of generality, assume $j>i$. Then,

$$
a_{i j}=a_{j i}=\sqrt{\alpha_{i} \alpha_{i+1} \ldots \alpha_{j-1}} \text {. }
$$

It follows that

$$
\left\|\mathbf{A}^{(0)}\right\|_{F}^{2} \geq 2 a_{i j}^{2}=2 \alpha_{i} \alpha_{i+1} \ldots \alpha_{j-1} .
$$

Therefore, for any numbers $\mu_{1}>\mu_{2}>\ldots \mu_{n}>0$ satisfying

$$
\frac{\sum_{1 \leq i<j \leq n}\left(\mu_{i}-\mu_{j}\right)^{2}}{2 \mu_{n}^{2} n}<\alpha_{i} \alpha_{i+1} \ldots \alpha_{j-1}
$$

the necessary conditions in Lemma 2 are not satisfied. This means that only using $(n-2)$ inerters (together with $n$ springs) is impossible to solve the MIEP for all arbitrarily given $s_{i}$, $i=1, \ldots, n$. In other words, $(n-1)$ inerters is the minimal number of inerters required to freely assign arbitrary values $0<\lambda_{1}<\lambda_{2}<\ldots \lambda_{n}$ as the eigenvalues of the $n$-DOF system. This completes the proof of necessity.

\subsection{Construction method}

The idea of proving the sufficiency of Theorem 4 is to choose $\delta_{i}$ and $\rho_{i}, i=1, \ldots, n$, separately, where $\delta_{i}, i=1, \ldots, n$, are intended to make the entries of $\mathbf{A}$ in (33) sufficiently small so that the MIEP is solvable, and $\rho_{i}, i=1, \ldots, n$, are obtained by solving the MIEP. This is now made more concrete in the following algorithm.

First step: obtain $\delta_{i}, i=1 \ldots, n$.

1. Choose $\epsilon_{1}>0$ so that

$$
\frac{\lambda_{i}}{\lambda_{i+1}}<1-\epsilon_{1}
$$

for all $i=1, \ldots, n-1$.

2. Find the unique solution with $0<\epsilon_{2}<1 / 2$ of

$$
2 \epsilon_{2}^{2}-\left(4-\epsilon_{1}\right) \epsilon_{2}+\epsilon_{1}=0
$$

which is equivalent to $\left(1-\epsilon_{2}\right)\left(1-2 \epsilon_{2}\right) /\left(1+\epsilon_{2}\right)=1-\epsilon_{1}$.

3. For an arbitrarily chosen $i$ set $\delta_{i}=0$ and let

$$
\delta_{j}=(n-1)^{2} / \epsilon_{2}^{2}-1
$$

for $j \neq i$.

Then, it can be checked that the condition $\max _{i} P_{i} \leq \epsilon_{2} \leq 1 / 2$ is satisfied as $\alpha_{i}<1$, $i=1, \ldots, n-1$, and that $(36)$ holds.

Second step: obtain $\rho_{i}, i=1, \ldots, n$.

The problem of finding $\rho_{i}, i=1, \ldots, n$ relies on numerically solving the MIEP problem. Specifically, there are three steps:

1. Construct matrix $\mathbf{A}$ according to (33) after obtaining $\delta_{i}, i=1, \ldots, n$; 
2. Obtain $d_{i}, i=1, \ldots, n$ by solving the MIEP;

3. Obtain $\rho_{i}=\frac{1+\delta_{i}}{d_{i}}, i=1, \ldots, n$.

In the second step, the method presented in [36] is employed. The idea is to solve the following nonlinear equations by using a one step Newton's method [36]

$$
f(\mathbf{d})=\left[\begin{array}{c}
s_{1}(\mathbf{d})-s_{1}^{*} \\
s_{2}(\mathbf{d})-s_{2}^{*} \\
\vdots \\
s_{n}(\mathbf{d})-s_{n}^{*}
\end{array}\right]=0,
$$

where $\mathbf{d}=\left[d_{1}, d_{2}, \ldots, d_{n}\right]$, and $s_{i}^{*}, i=1, \ldots, n$ denote the given numbers.

The following example is given to illustrate how to implement the proposed method.

Example 1. For a 5-DOF system, suppose $m_{1}=m_{2}=\ldots=m_{5}=100 \mathrm{~kg}$. Determine $b_{i}$ and $k_{i}, i=1, \ldots, 5$, such that the eigenvalues of the 5-DOF system are assigned as the following values

$$
\lambda=\left[\lambda_{1}, \ldots, \lambda_{5}\right]^{T}=[8.0808,52.8728,100.0000,223.8426,348.5371]^{T} .
$$

Following the method introduced in this section, since $s_{i}=1 / \lambda_{i}, i=1, \ldots, n$, we obtain $s$ as

$$
s=\left[s_{1}, \ldots, s_{5}\right]^{T}=\left[\begin{array}{lllll}
0.1238, & 0.0189, & 0.0100,0.0045,0.0029
\end{array}\right]^{T} .
$$

From (39), $\epsilon_{1}=0.0129$, and from (40), $\epsilon_{2}$ should be in the range $(0,0.0032)$. Hence, we choose $\epsilon_{2}=0.0031$. Since $(n-1)$ inerters are required, from (41), we can arbitrarily set one $\delta_{i}, i=1, \ldots, n$ as 0 , and set others as $\delta_{j}=1.7060 \times 10^{6}, j=1, \ldots, n$ and $j \neq i$. In this example, we set $\delta_{1}=0$, and then we obtain the inertances as $b_{1}=0, b_{2}=6.8240 \times 10^{8}$, $b_{3}=5.1180 \times 10^{8}, b_{4}=3.4120 \times 10^{8}$, and $b_{5}=1.7060 \times 10^{8}$.

Then, we obtain matrix $\mathbf{A}$ according to (33). After solving an MIEP, we obtain

$$
\mathbf{d}=\left[\begin{array}{llll}
0.1238, & 0.0189,0.0100, & 0.0045,0.0029
\end{array}\right]
$$

and $\rho_{1}=8.0808, \rho_{2}=9.0201 \times 10^{7}, \rho_{3}=1.7060 \times 10^{8}, \rho_{4}=3.8187 \times 10^{8}$, and $\rho_{5}=$ $5.9460 \times 10^{8}$. Finally, we obtain the spring stiffnesses as $k_{1}=4040.40, k_{2}=3.6080 \times 10^{10}$, $k_{3}=5.1180 \times 10^{10}, k_{4}=7.6375 \times 10^{10}$, and $k_{5}=5.9460 \times 10^{10}$.

Note that this method requires $(n-1)$ inerters which may not always be necessary. [For example, for Example 1, another solution to this problem is $b_{1}=b_{2}=b_{3}=b_{4}=0 \mathrm{~kg}$, $b_{5}=100 \mathrm{~kg}$, and $k_{1}=k_{2}=k_{3}=k_{4}=k_{5}=10 \mathrm{kN} / \mathrm{m}$.] Also the algorithm is conservative and may lead to large parameter values. Firstly, the algorithm ensures that the RHS in (36) is less than the minimum over $i$ of the LHS, whereas in order to satisfy the sufficient conditions of Lemma 1 it is only necessary to satisfy (36) individually for each $i$. Secondly, the ordering of the $s_{i}$ in Lemma 1 is not prescribed, so this is a further freedom which could be exploited. In particular if $\sigma$ denotes any permutation of the integers $1,2, \ldots, n$ and $\mu_{\sigma(i)}=1 / \lambda_{i}$ for $i=1, \ldots, n$ then $(36)$ is replaced by

$$
\frac{\lambda_{i+1}}{\lambda_{i}}>\frac{1+P_{\sigma(i+1)}}{\left(1-P_{\sigma(i+1)}\right)\left(1-2 P_{\sigma}(i)\right)}
$$


for $i=1, \ldots, n$. Thus it may be possible to satisfy (42) with smaller parameter values or a reduced number of inerters. It should be emphasise that the purpose of this paper was to prove the existence of solutions. For optimal design in practice, numerical optimization methods such as the quadratically convergent methods in [36], the LU decomposition methods in $[37,38]$ may usefully supplement the results of this paper. The problem of constructing explicit families of either isospectral or quasi-isospectral mass-chain systems with inerters is an interesting topic to be explored in the future.

\section{Conclusions}

This paper has studied the natural frequency assignment problem for mass-chain systems with inerters, where the adjacent masses in the mass-chain system are connected by a parallel arrangement of a spring and an inerter. The problem for mass-chain systems with given and fixed masses, whether it is possible to use only inerters and springs to realize any arbitrarily given real positive numbers as the natural frequencies of the mass-chain system was considered. A major result of the paper is that $n-1$ inerters and $n$ springs are necessary and sufficient to freely assign any arbitrarily given set of positive distinct numbers as the natural frequencies of $n$-DOF mass-chain systems. A constructive method of determining inertances and spring stiffnesses was proposed and verified by using a numerical example. It was also shown that multiple eigenvalues are possible, unlike conventional mass-chain systems without inerters whose natural frequencies are always simple and distinct. Some restrictions on the multiplicities that may occur were derived. In particular, it was shown that an eigenvalue of multiplicity $m$ may occur only if $n \geq 2 m-1$. Moreover, the inverse eigenvalue problem for continuous systems, presented as partial differential equations and infinite natural frequencies, was not studied in this paper, and would be considered in the future.

\section{Acknowledgment}

This work was supported in part by the National Natural Science Foundation of China under Grants 61603122 and 61374053, in part by the Natural Science Foundation of Jiangsu Province of China under Grant BK20160873, and in part by the Fundamental Research Funds for the Central Universities of China under Grant 2016B03814.

\section{References}

[1] M.C. Smith, Synthesis of mechanical networks: the inerter, IEEE Transactions on Automatic Control 47 (10) (2002) 1648-1662.

[2] M.Z.Q. Chen, C. Papageorgiou, F. Scheibe, F.-C. Wang, M.C. Smith, The missing mechanical circuit element, IEEE Circuits and Systems Magazine 9 (1) (2009) 10-26.

[3] M.C. Smith, F.-C. Wang, Performance benefits in passive vehicle suspensions employing inerters, Vehicle System Dynamics 42 (4) (2004) 235-257.

[4] Y. Hu, M.Z.Q. Chen, Z. Shu, Passive vehicle suspensions employing inerters with multiple performance requirements, Journal of Sound and Vibration 333 (2014) 2212-2225. 
[5] M.Z.Q. Chen, Y. Hu, C. Li, G. Chen, Performance benefits of using inerter in semiactive suspensions, IEEE Transactions Control Systems Technology 23 (4) (2015) 1571-1577.

[6] P. Li, J. Lam, K.C. Cheung, Control of vehicle suspension using an adaptive inerter, Proceedings of the Institution of Mechanical Engineers, Part D: Journal of Automobile Engineering (2015) 0954407015574808.

[7] F.-C. Wang, M.K. Liao, The lateral stability of train suspension systems employing inerters, Vehicle System Dynamics 48 (5) (2010) 619-643.

[8] A.Z. Matamoros-Sanchez, R.M. Goodall, Novel mechatronic solutions incorporating inerters for railway vehicle vertical secondary suspensions, Vehicle System Dynamics 53 (2) (2015) 113-136.

[9] F.-C. Wang, M.F. Hong, C.W. Chen, Building suspensions with inerters, Proceedings of the IMechE, Part C: Journal of Mech. Engineering Science 224 (8) (2010) 1605-1616.

[10] I.F. Lazar, S.A. Neild, D.J. Wagg, Using an inerter-based device for structural vibration suppression, Earthquake Engineering \& Structural Dynamics 43 (8) (2014) 1129-47.

[11] Y. Hu, M.Z.Q. Chen, Performance evaluation for inerter-based dynamic vibration absorbers, International Journal of Mechanical Sciences 99 (2015) 297-307.

[12] Y. Hu, M.Z.Q. Chen, S. Xu, Y. Liu, Semiactive inerter and its application in adaptive tuned vibration absorbers, IEEE Transactions on Control Systems Technology 25 (1) (2017) 294-300.

[13] P. Brzeski, E. Pavlovskaia, T. Kapitaniak, P. Perlikowski, The application of inerter in tuned mass absorber, International Journal of Non-Linear Mechanics 70 (2015) 20-29.

[14] Y. Hu, M.Z.Q. Chen, Z. Shu, L. Huang, Analysis and optimisation for inerter-based isolators via fixed-point theory and algebraic solution, Journal of Sound and Vibration 346 (2015) 17-36.

[15] X. Dong, Y. Liu, M.Z.Q. Chen, Application of inerter to aircraft landing gear suspension, Proceedings of the 34th Chinese Control Conference, Hangzhou, China, 2015, pp. 20662071.

[16] M.Z.Q. Chen, M.C. Smith, Restricted complexity network realizations for passive mechanical control, IEEE Transactions on Automatic Control 54 (10) (2009) 2290-2301.

[17] M.Z.Q. Chen, K. Wang, Y. Zou, J. Lam, Realization of a special class of admittances with one damper and one inerter for mechanical control, IEEE Transactions on Automatic Control 58 (7) 1841-1846.

[18] M.Z.Q. Chen, K. Wang, Y. Zou, G. Chen, Realization of three-port spring networks with inerter for effective mechanical control, IEEE Transactions on Automatic Control 60 (10) (2015) 2722-2727. 
[19] K. Yamamoto, M.C. Smith, Bounded disturbance amplification for mass chains with passive interconnection, IEEE Transactions on Automatic Control 61 (6) (2016) 15651574.

[20] N. Jalili, N. Olgac, Multiple delayed resonator vibration absorbers for multi-degree-offreedom mechanical structures, Journal of Sound and Vibration 223 (4) 567-585.

[21] G.M.L. Gladwell, Inverse Problems in Vibration, Second Edition, Springer Science \& Business Media, 2005.

[22] S.P. Beeby, M.J.Tudor, N.M. White, Energy harvesting vibration sources for microsystems applications, Measurement Science and Technology 17 (12) (2006) R175-R195.

[23] Y. Yu, N.G. Naganathan, R.V. Dukkipati, A literature review of automotive vehicle engine mounting systems, Mechanism and Machine Theory 36 (1) (2001) 123-142.

[24] J.E. Mottershead, Y.M. Ram, Inverse eigenvalue problems in vibration absorption: Passive modification and active control, Mechanical Systems and Signal Processing 20 (1) (2006) 5-44.

[25] Y. Liang, H. Yamaura, H. Ouyang, Active assignment of eigenvalues and eigensensitivities for robust stabilization of friction-induced vibration, Mechanical Systems and Signal Processing 90 (2017) 254-267.

[26] P. Olsson, P. Lidström, Inverse structural modification using constraints, Journal of Sound and Vibration 303 (2007) 767-779.

[27] H. Ouyang, J. Zhang, Passive modifications for partial assignment of natural frequencies of mass-spring systems, Mechanical Systems and Signal Processing 50 (2015) 214-226.

[28] P. Nylen, F. Uhlig, Inverse eigenvalue problem associated with spring-mass systems, Linear Algebra and its Applications 254 (1) (1997) 409-425.

[29] M.Z.Q. Chen, Y. Hu, L. Huang, G. Chen, Influence of inerter on natural frequencies of vibration systems, Journal of Sound and Vibration 333 (7) (2014) 1874-1887.

[30] M.T. Chu, G.H. Golub, Inverse Eigenvalue Problems: Theory, Algorithms, and Applications, Oxford Science, New York, 2005.

[31] F.R. Gantmacher, The Theory of Matrices. Vols. 2. Translated by K. A. Hirsch. Chelsea Publishing Co., New York, 1959.

[32] J.H. Wilkinson, The Algebraic Eigenvalue Problem. Oxford: Clarendon Press, 1965.

[33] G.N. de Oliveira, On the multiplicative inverse eigenvalue problem, Canadian Mathematical Bulletin 15 (2) (1972) 189-173.

[34] S. Xu, On the necessary conditions for the solvbility of algebraic inverse eigenvalue problems, Journal of Computional Mathematics 10 (1) (1992) 93-97. 
[35] L. Li, On solvability of inverse eigenvalue problems with Hermitian matrices, Linear Algebra and its Applications 253 (1) (1997) 89-101.

[36] S. Friedland, J. Nocedal, M.L. Overton, The formulation and analysis of numerical methods for inverse eigenvalue problems, SIAM Journal on Numerical Analysis 24 (3) (1987) 634-667.

[37] H. Dai, Z. Bai, and Y. Wei. On the solvability condition and numerical algorithm for the parameterized generalized inverse eigenvalue problem, SIAM Journal on Matrix Analysis and Applications 36 (2) (2015) 707-726.

[38] H. Dai and Z. Bai, On smooth LU decompositions with applications to solutions of nonlinear eigenvalue problems, J. Comput. Math. 28 (2010) 745-766. 